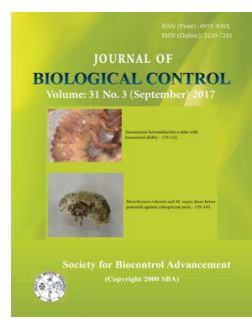

\title{
Research on Coccinellidae (Coleoptera) fauna in Mazandarn province, Iran
}

\author{
KATAYOUN PAHLAVAN YALI ${ }^{1}$, SHAHROKH PASHAI RAD ${ }^{1}$, MEHDI ZARE KHORMIZI ${ }^{*}$, ZAHRA MOJIB \\ HAGH GHADAM ${ }^{3}$, MINOO HEIDARI LATIBARI ${ }^{4}$ and GUY HANLY ${ }^{5}$ \\ ${ }^{I}$ Faculty of Biological Sciences, Shahid Beheshti University, Tehran, Iran \\ ${ }^{2}$ Young Researchers and Elite Club, Yazd Branch, Islamic Azad University, Yazd, Iran \\ ${ }^{3}$ Guilan Agricultural and Natural Resources Research and Education Center, Rasht, Guilan, Iran \\ ${ }^{4}$ Department of Plant Protection, Faculty of Agriculture, Ferdowsi University of Mashhad, Mashhad, Iran \\ ${ }^{5}$ Northern Plains Entomology, Minot, North Dakota \\ ${ }^{*}$ Corresponding author E-mail: zare7002@gmail.com
}

ABSTRACT: Ladybirds beetles belonging to the family Coccinellidae, Order Coleoptera, play an important role in pest control. The aim of this research was to explore, identify and characterize the coccinellid fauna of the Mazandaran Province (Iran). Coccinellid beetles were collected during 2013- 2015 from different Agriculture Stations with a wide range of agricultural lands and plants. A total of 21 species in 15 genera and 4 subfamilies were identified.

1. Coccinella septempunctata (Linnaeus, 1758)

2. Adalia bipunctata (Linnaeus, 1758)*

3. Harmonia quadripunctata (Pontoppidan, 1763) *

4. Haippodemia variegate (Goeze, 1777)

5. Oenopia congolobata (Linnaeus, 1758)

6. Propyleaquatuor decempunctata (Linnaeus, 1758)

7. Adalia decempunctata (Linnaeus, 1758)

8. Vibidia duodecimguttata (Poda, 1761) *

9. Psylloboraviginti duopunctata (Linnaeus, 1758)

10. Chilocorus bipustulatus (Linnaeus, 1758)

11. Exochomus nigromaculatus (Goeze, 1777)

12. Platynaspis luteorubra (Redtenbacher, 1843)*

13. Nephusbi punctatus (Kugelann, 1794)*

14. Nephus qadrimaculatus (Herbst, 1783)

15. Scymnus subvillosus (Goeze, 1777)

16. Scymnus frontalis (Fabricius, 1787)*

17. Scymnus apetzy (Mulsant, 1846)

18. Scymnus impexus (Mulsant, 1850)*

19. Stethorus punctilum (Weise, 1891)*

20. Stethorus gilvifrons (Mulsant 1850)*

21. Serangium montazeri (Montazeri, 1994)*

Nine species that are marked by *are new records for MazandaranCoccinellid fauna.

KEY WORDS: Ladybird, New record, Mazandaran, Insect

(Article chronicle: Received: 29-06-2017; Revised: 12-09-2017; Accepted: 30-09-2017)

\section{INTRODUCTION}

The Family Coccinellidae, which are known as Ladybugs and ladybird beetles, belong to the superfamily Cucujoidea, order Coleoptera. This family is the largest in the recently characterized superfamily Coccinellidae (Robertson et al., 2015). The color patterns of ladybirds is surprisingly variable, which can range from a reddish or yellowish to black background with dark and light spots, 
respectively, or to a brown background with light spots. Ladybirds also have various diets, and although they are mainly predators, some are phytophagous, and are serious economic pests for crops (Shaefer, 1983). Most members of this family, are beneficial insects (William, 2002), With many species preying on herbivorous pests such as aphids or scale insects. Most coccinellids lay their eggs directly on aphids and scale insects colonies, hence they ensure that their larvae have access to the necessary food source (Montazer and Mosaddegh, 1995). Some species, such as the subfamily Epilachninae, is phytophagous and have undesirable effects causing damage to various crops such as potatoes and beans Their numbers are decreased with activity of parasitoid wasps.

\section{MATERIALS AND METHODS}

Specimens were collected from 15 different Stations in Mazandaran Province Iran (36 33' 56.16" N, 53 3' 31.68" E) during 2013-2015. Collecting methods included aspirator, white dish and direct hand picking from various plants. The specimens were sent to the Biosystematic laboratory of Shahid Beheshti University for identification. All species were stored in $70 \%$ ethanol and were identified using available keys (Reimundo and Harten, 2000) in few cases, genitalic dissections were carried out by using $10 \% \mathrm{KOH}$ and permanent slides were made by Hoyer solution. All identified samples were confirmed by Dr. Oldrich Nedved at university of south Bohemia in Czech Republic.

Table 1. List of the sampling localities in west of Mazandaran Province

\begin{tabular}{|c|l|l|l|}
\hline Number & Locations & Coordinate of locations & Altitude \\
\hline 1 & Ramsar & $36^{\circ} 90 \mathrm{~N}-50^{\circ} 67^{\prime} \mathrm{E}$ & -8 \\
\hline 2 & Javaherdeh & $36^{\circ} 91 \mathrm{~N}-50^{\circ} 61^{\prime} \mathrm{E}$ & 1768 \\
\hline 3 & Tnekabon & $36^{\circ} 81 \mathrm{~N}-50^{\circ} 87^{\prime} \mathrm{E}$ & -14 \\
\hline 4 & GhaleGardan & $36^{\circ} 75 \mathrm{~N}-50^{\circ} 84^{\prime} \mathrm{E}$ & 45 \\
\hline 5 & Falakdeh & $36^{\circ} 61 \mathrm{~N}-50^{\circ} 92^{\prime} \mathrm{E}$ & 923 \\
\hline 6 & EshkevarMahaleh & $36^{\circ} 64 \mathrm{~N}-50^{\circ} 72^{\prime} \mathrm{E}$ & 1395 \\
\hline 7 & Abbas Abad & $36^{\circ} 72 \mathrm{~N}-51^{\circ} 11^{\prime} \mathrm{E}$ & -20 \\
\hline 8 & Valasht Lake & $36^{\circ} 49 \mathrm{~N}-51^{\circ} 30^{\prime} \mathrm{E}$ & 774 \\
\hline 9 & Chaloos & $36^{\circ} 49 \mathrm{~N}-51^{\circ} 30^{\prime} \mathrm{E}$ & 80 \\
\hline 10 & KalarDasht & $36^{\circ} 64 \mathrm{~N}-51^{\circ} 40^{\prime} \mathrm{E}$ & 1343 \\
\hline 11 & Marzan Abad & $36^{\circ} 44 \mathrm{~N}-51^{\circ} 29^{\prime} \mathrm{E}$ & 519 \\
\hline 12 & ArbehKaleh & $36^{\circ} 86 \mathrm{~N}-50^{\circ} 67^{\prime} \mathrm{E}$ & 312 \\
\hline 13 & Garsmasar & $36^{\circ} 81 \mathrm{~N}-50^{\circ} 56^{\prime} \mathrm{E}$ & 2205 \\
\hline 14 & Nashtarood & $36^{\circ} 78 \mathrm{~N}-50^{\circ} 94^{\prime} \mathrm{E}$ & -22 \\
\hline 15 & Salman Shahr & $36^{\circ} 70 \mathrm{~N}-51^{\circ} 19^{\prime} \mathrm{E}$ & -10 \\
\hline
\end{tabular}

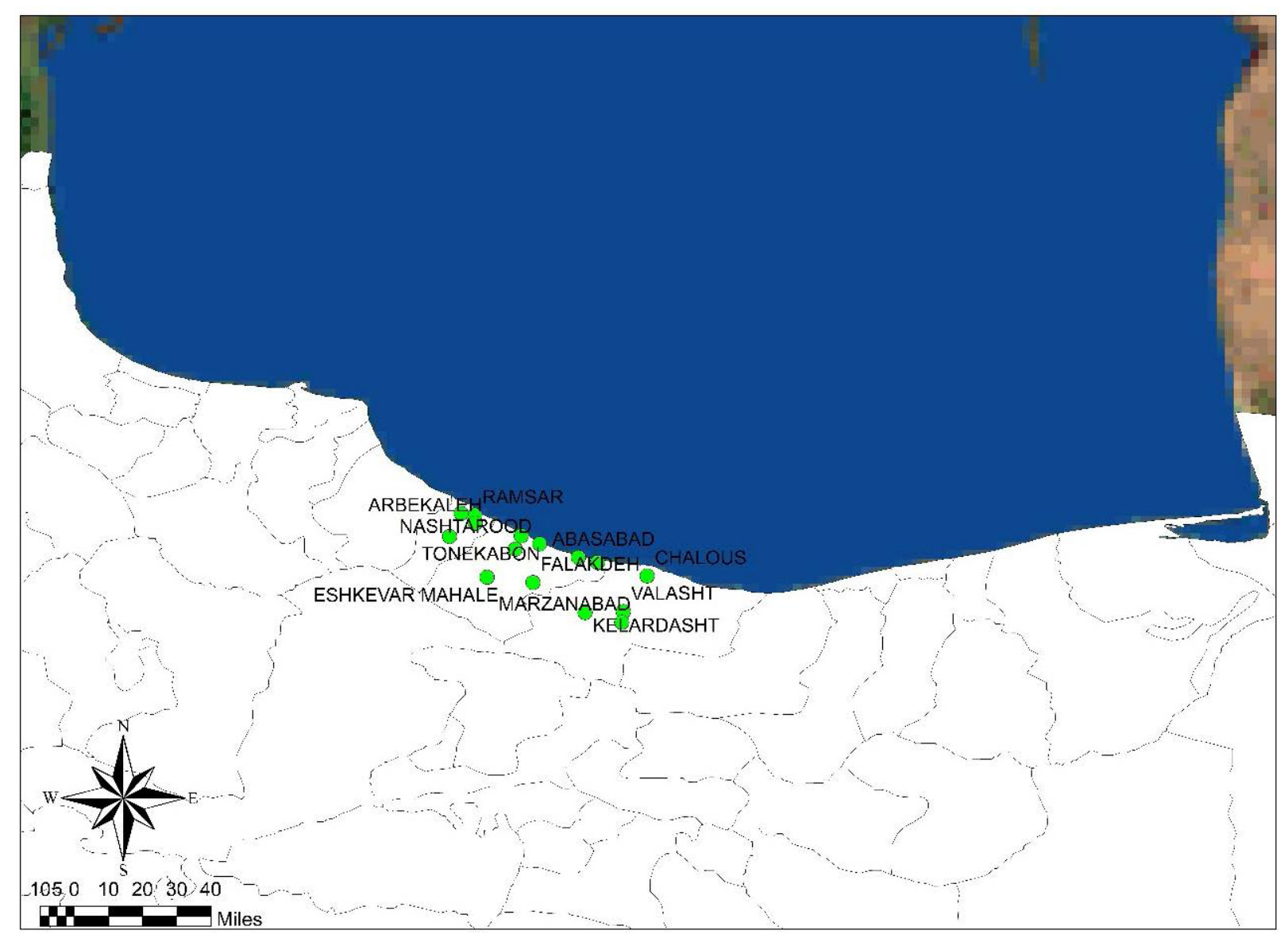

Fig. 1. Drawn map of the stations. 


\section{RESULTS AND DISCUSSION}

A total of 21 species belong to 15 genera and 4 subfamilies were identified. Identified species are listed as below:

\section{ACKNOWLEDGMENTS}

Thanks to Dr. Oldrich Nedved, Associate Professor at University of South Bohemia, Czech Republic for identification of ladybirds species. This research was a part of MSc studies of the first author at Shahid Beheshti University, Tehran, Iran.
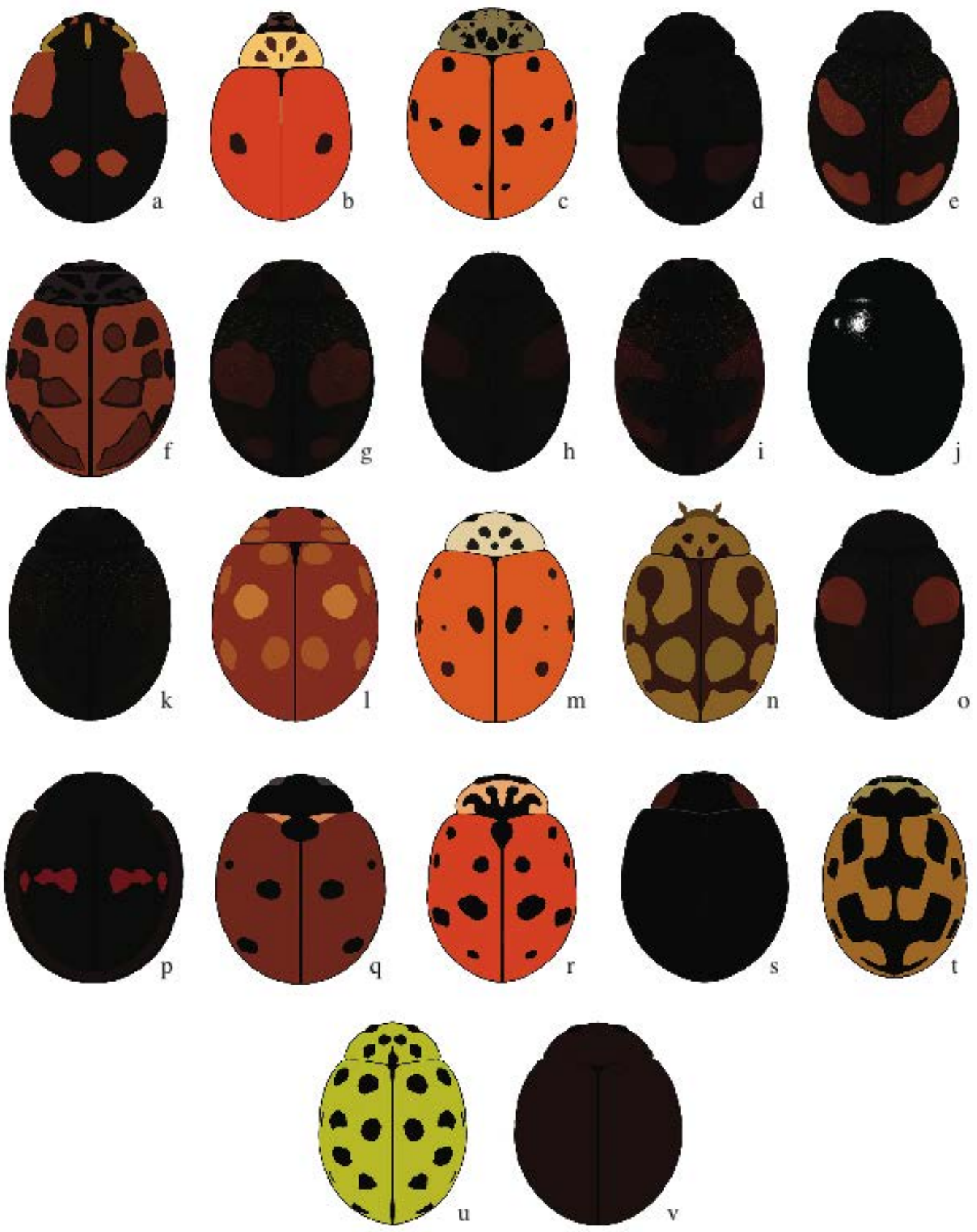

Fig. 2. a-b) Adalia bipunctata (Linnaeus, 1758), c) Harmonia quadripunctata (Pontoppidan, 1763), d) Nephus bipunctatus (Kugelann, 1794), e) Nephus qadrimaculatus (Herbst, 1783), f) Oenopia congolobata (Linnaeus, 1758), g) Platynaspis luteorubra (Redten Bacher, 1843), h) Scymnus frontalis (Fabricius, 1787), i) Scymnus subvillosus (Goeze, 1777), j) Stethorus gilvifrons (Mulsant 1850), k) Stethorus punctilum (Weise, 1891), l) Vibidiaduo decimguttata (Poda, 1761), m-n) Adalia decempunctata (Linnaeus, 1758), o) Scymnus apetzi (Mulsant, 1846), p) Chilocorus bipustulatus (Linnaeus, 1758), q) Coccinella septempunctata (Linnaeus, 1758), r) Hippodamia variegata (Goeze, 1777), s) Parexochomus nigromaculatus (Goeze, 1777), t) Propyleaquatuor decimpunctata (Linnaeus, 1758), u) Psyllobora vigintiduo punctata (Linnaeus, 1758), v) Serangium montazerii (Montazeri, 1994) All images have been scaled to one standard size, refer to species description for actual size. 
Table 2. Collected species and Host plant in north of Iran

\begin{tabular}{|c|c|}
\hline Species & Host plant \\
\hline Adalia bipunctata (Linnaeus, 1758) & Juglans regia $\mathrm{L}$, Urtica folium \\
\hline Adalia decempunctata (Linnaeus, 1758) & $\begin{array}{l}\text { U. folium, J. regia, Citrus sp, Punica granatum, } \\
\text { Cirsium vulgare }\end{array}$ \\
\hline Chilocorus bipustulatus (Linnaeus, 1758) & Citrus sp. \\
\hline Coccinella septempunctata (Linnaeus, 1758) & $\begin{array}{l}\text { Trifolium } \mathrm{sp}, \text { Phlomis persica, Eryngium planum, } \\
\text { Galium aparine L, U. folium, C. vulgare, Citrus } \\
\text { sp. }\end{array}$ \\
\hline Harmonia quadripunctata (Pontoppidan,1763) & U. folium \\
\hline Haippodemia variegate (Goeze, 1777) & U. folium \\
\hline Nephus bipunctatus (Kugelann, 1794) & C. vulgare \\
\hline Nephus qadrimaculatus (Herbst, 1783) & Citrus sp. \\
\hline Oenopia congolobata (Linnaeus, 1758) & $\begin{array}{l}\text { U. folium, C. vulgare, Trifolium sp, Verbascum } \\
\text { thapsus }\end{array}$ \\
\hline Propylea quatuordecimpunctata (Linnaeus, 1758) & J. regia, U. folium, Citrus sp. \\
\hline Psyllobora vigintiduopunctata (Linnaeus, 1758) & G. aparine $\mathrm{L}$ \\
\hline Parexochomus nigromaculatus (Goeze, 1777) & U. folium, C. vulgare, \\
\hline Platynaspis luteorubr (Redtenbacher,1843) & Citrus sp. \\
\hline Scymnus subvillosus (Goeze, 1777) & P. granatum, Citrus sp. \\
\hline Scymnus frontalis (Fabricius, 1787) & P. granatum, Citrus sp. \\
\hline Scymnus apetzi (Mulsant, 1846) & C. vulgare \\
\hline Scymnus impexus (Mulsant, 1850) & Citrus sp. \\
\hline Stethorus punctilum (Weise, 1891) & Citrus sp. \\
\hline Stethorus gilvifrons (Mulsant 1850) & Citrus sp, J. regia \\
\hline Serangium montazerii (Montazeri, 1994) & Citrus sp. \\
\hline Vibidia duodecimguttata (Poda, 1761) & Morus sp., Citrus sp. \\
\hline
\end{tabular}

\section{REFERENCES}

Alizadeh MS, Mossadegh MS, Esfandiari M. 2013. Natural enemies of Maconellicoccus hirsutus (Green) (Hemiptera: Pseudococcidae) and their population fluctuations in Ahvaz, southwest of Iran. J Crop Prot. 2(1):1 3-21.

Biranvand A, Jafari R, Zare Khormizi M. 2014. Diversity and distribution of Coccinellidae (Coleoptera) in Lorestan province, Iran. Biodiversity J. 5(1): 3-8.

Ebrahimi S, ModarresAwal M, Karimi J, Fekrat L, Nedved O. 2014. Two new records of ladybirds (Col.: Coccinellidae) for the Iranian beetle fauna. $J$ Entomol Soc Iran 34: 11-12.

Farsi A, Kocheili F, Mossadegh MS, Rasekh A, Tavoosi M. 2014. Natural enemies of the currant lettuce aphid, Nasonovia ribisnigri (Mosely) (Hemiptera: Aphididae) and their population fluctuations in Ahvaz, Iran. J Crop Prot. 3(4): 487-497.

Hunt T, Bergsten J, Levkanicova Z, Papadopoulou A, John OS, Wild R, Hammond PM, Ahrens D, Balke M, Caterino MS, Gomez-Zurita J, Ribera I, Barraclough TG, Bocakova M, Bocak L, Vogler AP. 2007. A comprehensive phylogeny of beetles reveals the evolutionary origins of a superradiation. Science 318: 1913-1916. Crossref PMid:18096805

Mehrnejad MR, Jalali MA, Mirzaei R. 2011. Abundance and biological parameters of psyllophagous coccinellids in pistachio orchards. J Appl Ent. 135(9): 673-681. Crossref

Michaud JP.2004. Natural mortality of Asian citrus psyllid, Diaphorina citri (Homoptera: Psyllidae) in central 
Florida. Science Direct. Biol Control 29: 260-269. Crossref

Montazeri MM, Mossadegh MS. 1995. The coccinellids (Coleoptera) fauna of Gorgan plain and Gonbad Kavus, p: 325. In: Proceeding of the 12Th plant protection congress of Iran 2- 7 September 1995, Karaj, Iran.

Raimundo AC, Harten AV. 2000. An annotated checklist of the Coccinellidae (Insecta: Coleoptera) of Yemen. Fauna Arabia 18: 211-243.

Robertson J, Sliplipinski A, Moulton M, Shockley F et al. 2015. Phylogeny and classification of Cucujoidae and recognition of a new superfamily Coccinelloidae (Coleoptera: Cucujiformia). Syst Entomol. 40(4): 745-778. Crossref

Salehi T, Mehrnejad MR, Pashaei S. 2013. Diversity pattern of adult ladybird (Coleoptera: Coccinellidae) communities on pistachio trees in southern parts of Iran in different months. Zool Ecol. 23(4): 286-292. Crossref
Shaefer PW. 1983. Natural enemies and host plants of species in the Epilachninaea world list. Agric. Exp. Station, Univ. Delaware, Bull. 445: 1-42.

Smirnoff WA. 1956. Observation sur les prédateurset parasites des cochenillesnuisibles du Maroc et surleursennemis. Ministère de l'Agriculture et des Forêts, Service de la Défense des Végétaux 11: 1-60.

William FL. 2002. Lady Beetles. Ohio State University Extension Fact Sheet, Horticulture and Crop Science. Division of Wildlife, 2021 Coffey Rd. Columbus, Ohio, $857 \mathrm{pp}$.

Zare Khormizi M, Ostovan H, Fallahzadeh M. 2014. Status of Ladybirds (Coleoptera:Coccinellidae) in Khatam County (Yazd Province) and the first report of Hyperaspis reppensis (Herbst, 1783) for Iranian Fauna. J Biol Control 28:126-131 